\title{
Cystic Fibrosis Pulmonary Exacerbation
}

National Cancer Institute

\section{Source}

National Cancer Institute. Cystic Fibrosis Pulmonary Exacerbation. NCI Thesaurus. Code C121562.

An acute episode of worsening pulmonary symptoms related to cystic fibrosis. 